\title{
PHYSICS, LASERS AND THE NOBEL PRIZE
}

- Antigone Marino - DOl: https://doi.org/10.1051/epn/2019205

I Institute of Applied Sciences and Intelligent Systems, National Research Council - c/o Physics Department - University of Naples Federico II, Italy

Like every year, we have been waiting for the announcement of the most important award for natural sciences, the Nobel Prize in Physics. Perhaps it is since 2013, the year in which the award was given for the discovery of the Higgs boson, that the Nobel Prize has great media visibility. The public, scientific or otherwise, is waiting to know who will be awarded. 
W e also know that, following the announcements, social media will be responsible for making the general public understand who and especially which discovery has been rewarded. The Nobel Prize in Physics 2018 was awarded "for groundbreaking inventions in the field of laser physics" with one half to Arthur Ashkin "for the optical tweezers and their application to biological systems" [1], the other half jointly to Gérard Mourou and Donna Strickland "for their method of generating high-intensity, ultra-short optical pulses" [2]. Since 1964, when Charles Hard Townes, Nicolay Gennadiyevich Basov and Aleksandr Mikhailovich Prokhorov were awarded "for fundamental work in the field of quantum electronics, which has led to the construction of oscillators and amplifiers based on the maser-laser principle" [3], many Nobel prizes in physics have seen the laser as a best actor or supporting actor. Even this year what seems to have been awarded is not only the excellent work of three scientists, but also the human enterprise of science. Making a quick overview of the physics Nobel Prizes history, we can find the laser in 1971 with Dennis Gabor "for his invention and development of the holographic method" [4]. In 1981 with Nicolaas Bloembergen and Arthur Leonard Schawlow "for their contribution to the development of laser spectroscopy" [5], together with Kai M. Siegbahn "for his contribution to the development of high-resolution electron spectroscopy" [6]. In 1997 Steven Chu, Claude Cohen-Tannoudji and William D. Phillips were awarded "for development of methods to cool and trap atoms with laser light" [7]. In 2005 Roy J. Glauber won "for his contribution to the quantum theory of optical coherence" [8] together with John L. Hall and Theodor W. Hänsch "for their contributions to the development of laser-based precision spectroscopy, including the optical frequency comb technique" [9]. Even the 2017 prize, that will be remembered as the gravitational waves award, it actually went to Rainer Weiss, Barry C. Barish and Kip S. Thorne "for decisive contributions to the LIGO detector and the observation of gravitational waves" [10], one of the biggest laser based facility in the word. Surely from this excursus I may have left out some years, some Nobel Prizes, perhaps not in physics but in chemistry or medicine, who have had the laser as an actor. However, the mentioned awards already give a measure of how much laser technology has been treading the stage of science for more than 50 years.

And if in science they rode the stage of success, in every day life they went among the people. Just think of the countless laser applications such as medical ones, eye surgery, cancer treatment, or industrial applications such as laser etching, welding and drilling. Even the barcode readers, CD players, car distance sensors. This list could last for the full length of the article, it is sure.

The way a laser works is easy to understand. When the electrons in atoms in special glasses, crystals, or gases

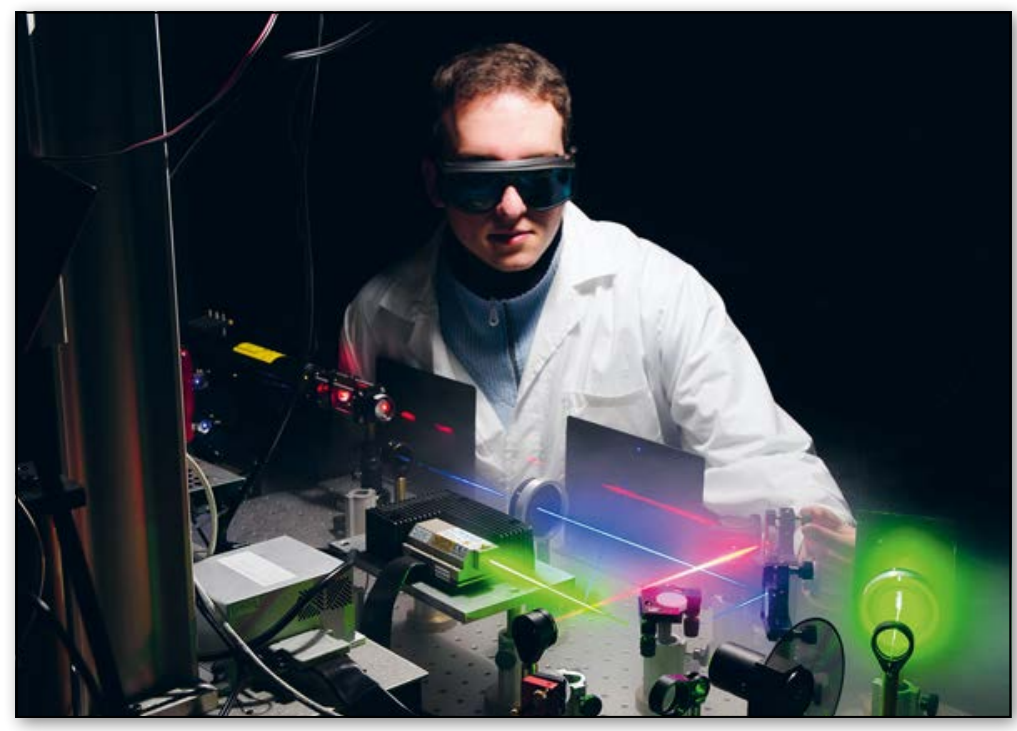

absorb energy they become excited. The excited electrons move from a lower-energy orbit to a higher-energy orbit. When they return to the ground state, the electrons emit photons. Laser light is different from normal light for two reasons: these emitted photons are all at the same wavelength, which means that the generated light is coherent (figure on p. 26); the latter is that laser light is directional. Whereas a laser generates a very tight beam, a flashlight produces light that is diffuse.

There are many ways to control laser light. For instance, using different materials which leads to different electron transitions, thus different energies and wavelengths. Otherwise one can act on the collimation design, or different amplification systems in order to create more powerful lasers. Having control of the laser properties opens up new possibilities for probing and manipulating matter. That is how optical tweezers have been developed, allowing Arthur Ashkin to win the Nobel Prize for 2018.

Optical tweezers use a highly focused laser beam to provide an attractive or repulsive force to physically hold and move microscopic objects (figure 1). The greater the laser power control is, the better the control in the manipulation of objects is. In 1986, Ashkin used the optical tweezers technique to trap living bacteria just illuminating them with lasers. In this EPN special issue we asked Antonio Sasso and his group to show us the last discoveries in the field of optical tweezers since this technique's invention.

Sometimes, what is needed is to have not only the laser power control, but also its pulse frequency. In some extreme cases what we might want is to have a single short burst. This was a very difficult task for scientists. The creation of a short and ultra-powerful impulse was not easy, often it involved the destruction of the material used to amplify the light and generate the laser light itself. This problem was solved by Gérard Mourou and Donna Strickland in 1985 . They proposed a four steps setup: first, they created standard laser pulses; then, they stretched $\triangle$ FIG. 1:

Force sensing and optical tweezers realized probing different wavelength, (c) Sebastiano Vasi
4 P26: Laser light is coherent, all emitted photons are all at the same wavelength, thus same colour. 


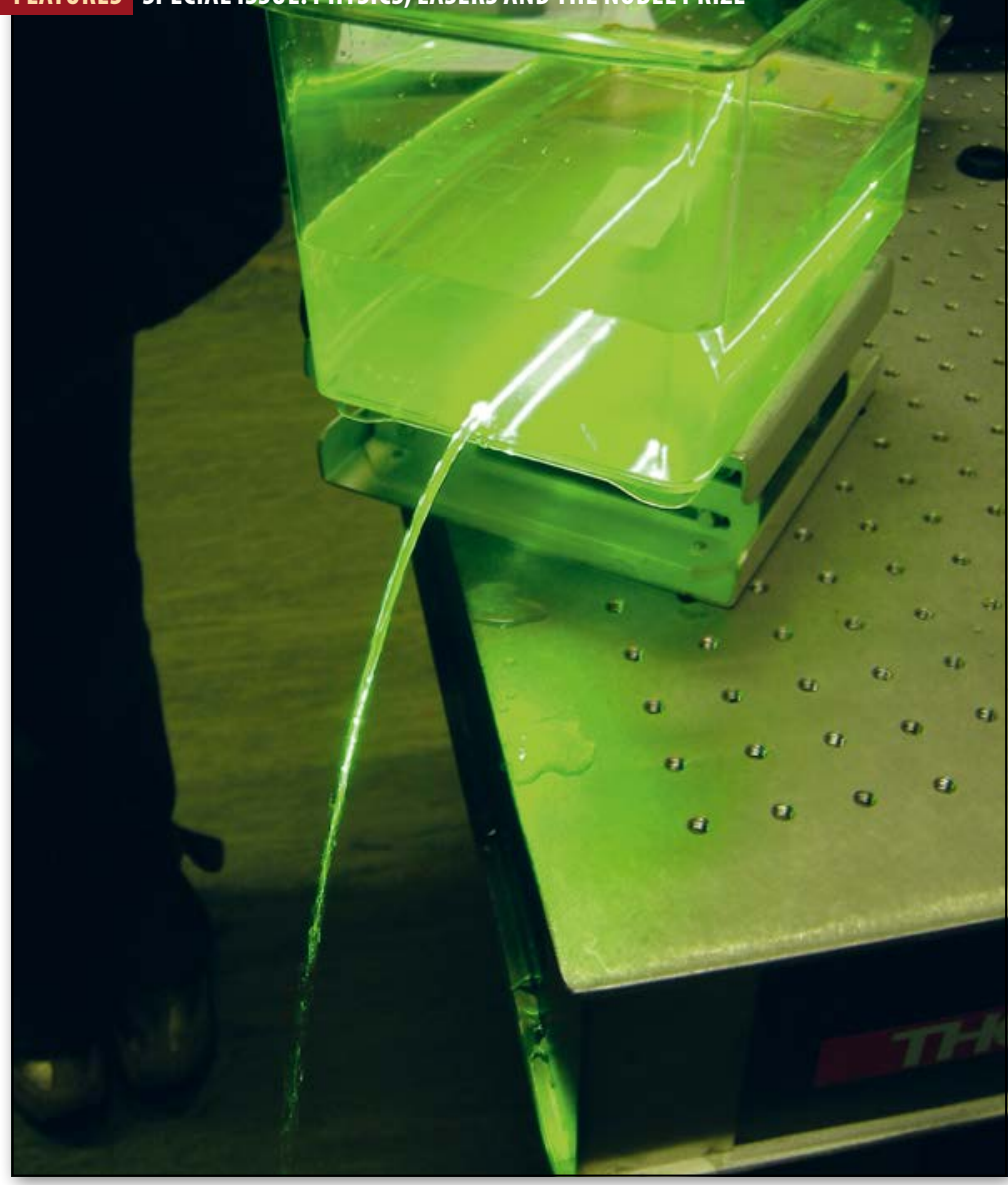

$\triangle F \mid$ G.2: the pulses in time, which reduces their peak power and

Laserlight makes them less destructive; next, they amplified the travelling through a stream of water flowing from a pipe (the John Tyndall's experiment) (c) Antigone Marino time-stretched, reduced-power pulses; and finally, they compressed the now-amplified pulses in time. The new technique, named chirped pulse amplification, became a standard for high-intensity lasers. In this EPN issue we asked Giulio Cerullo and his research group to review the capability of lasers to generate light pulses of incredibly short duration, from a few femtoseconds down to a few tens of attoseconds.

v FIG.3: The purpose of this EuroPhysics News's special issue Donna Strickland receiving the Nobel Prize in Stockholm. Picture by Alexander Mahmoud (C) Nobe Media AB 2018 and daily life. In the coming decades we will see a growing request and development of large facilities based on laser light, like the European X-Ray Free-Electron Laser Facility (European XFEL) in Germany or the Extreme Light Infrastructure (ELI) in the Czech Republic, in Hungary and in Romania. That's why we invited Victor Zamfir to write a feature on ELI, the laser facility that aims to host the most intense beamline system worldwide.

The scientific research produced by lasers is terrific. As a scientist in love with outreach activities, let me also mention the faces of the public when I show Tyndall's experiment (figure 2), how the laser light gets trapped in a gush of water acting as an optical fiber, or the fun of children when playing in a laser maze that even Lupin III could not escape. And that's why we have invited Christopher Holmes and his colleagues from the Southampton Optoelectronics Research Centre to introduce us to their famous light show.

The 2018 Nobel Prize in Physics has a strong historic importance because of Donna Strickland becoming the first woman to win a physics Nobel Prize in more than 50 years (figure 3). This issue ends with a beautiful opinion by Elizabeth Rogan, CEO of the Optical Society, who carefully describes the importance of this event not for women in science, but for science.

We hope that the reading of this issue will enlighten you, of coherent light, laser light!

\section{About the Author}

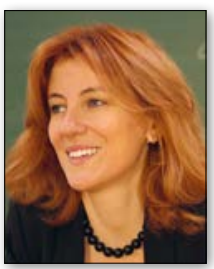

Antigone Marino is researcher at the Institute of Applied Sciences and Intelligent Systems of the Italian National Research Council in Naples, Italy. Her research activities are concentrated on the study of soft matter optics. She is in the OSA Foundation Board of Directors, topical editor of the Journal of the Optical Society of America A, of Euro Physics News and of Giornale di Fisica.

\section{References}

[1] A. Ashkin, J. M. Dziedzic, J. E. Bjorkholm, and Steven Chu, Opt. Lett. 11, 288 (1986)

[2] D. Strickland, G. Mourou, Opt. Comm. 56, 219 (1985).

[3] A. L. Schawlow and C. H. Townes, Phys. Rev. 112, 1940 (1958).

[4] D. Gabor, Nature 161, 777 (1948).

[5] N. Bloembergen, Rev. Mod. Phys. 54, 685 (1982)

[6] K. Siegbahn, N. Kholine and G. Golikov, Nuclear Instruments and Methods in Physics Research Section A 384, 56 (1997).

[7] W. D. Phillips, Reviews of Modern Physics, Vol. 70, No. 3 (1998).

[8] R. J. Glauber, Phys. Rev. 130, 2529 (1963)

[9] T. Hänsch, Nature Photonics 5, 193 (2011)

[10] B.P. Abbott et al. (LIGO Scientific Collaboration and Virgo Collaboration), Phys. Rev. Lett. 116, 061102 (2016). 